\title{
Bounds for the blow-up time of a porous medium equation with weighted nonlocal source and inner absorption terms
}

Yunde Shen ${ }^{1}$ and Zhong Bo Fang ${ }^{2^{*}}$ (1)

\section{"Correspondence:}

fangzb7777@hotmail.com

${ }^{2}$ School of Mathematical Sciences,

Ocean University of China, Qingdao,

P.R. China

Full list of author information is

available at the end of the article

\begin{abstract}
We investigate the blow-up phenomena for a porous medium equation with weighted nonlocal source and inner absorption terms subject to null Dirichlet boundary condition. Based on a modified differential inequality technique, we establish some sufficient conditions to guarantee the existence of non-global solutions to the model and also derive the upper bounds for the blow-up time. Moreover, the lower bounds for the blow-up time are obtained under some appropriate measure in the whole-dimensional space $(N \geq 1)$.
\end{abstract}

MSC: 35K59; 35B30; 35B40

Keywords: Porous medium equation; Weighted nonlocal source; Inner absorption; Bounds for the blow-up time

\section{Introduction}

We consider the porous medium equation with weighted nonlocal source and inner absorption terms

$$
u_{t}=\Delta u^{m}+a(x) u^{p} \int_{\Omega} u^{q} d x-u^{s}, \quad(x, t) \in \Omega \times\left(0, t^{*}\right),
$$

subject to null Dirichlet boundary and initial conditions

$$
\begin{aligned}
& u(x, t)=0, \quad(x, t) \in \partial \Omega \times\left(0, t^{*}\right), \\
& u(x, 0)=u_{0}(x) \geq 0, \quad x \in \Omega,
\end{aligned}
$$

where $\Omega \subset \mathbb{R}^{N}(N \geq 1)$ is a bounded region with smooth boundary $\partial \Omega, m, p, q>0, s \geq 1$. $t^{*}$ is a possible blow-up time when blow-up occurs, otherwise $t^{*}=+\infty$. The weight function $a(x) \in C^{1}(\Omega) \cap C^{0}(\bar{\Omega})$ satisfies

$\left(a_{1}\right) a(x) \geq a>0$ for all $x \in \bar{\Omega}$, where $a$ is a positive constant, or

$\left(a_{2}\right) a(x)>0, x \in \Omega$, and $a(x)=0, x \in \partial \Omega$.

Moreover, the initial data $u_{0}(x)$ is a positive $C^{1}$-function which satisfies a compatibility condition. Therefore, by the parabolic theory, it follows that the local weak solution to

(c) The Author(s) 2018. This article is distributed under the terms of the Creative Commons Attribution 4.0 International License (http://creativecommons.org/licenses/by/4.0/), which permits unrestricted use, distribution, and reproduction in any medium, provided you give appropriate credit to the original author(s) and the source, provide a link to the Creative Commons license, and indicate if changes were made. 
problem (1)-(3) exists uniquely, and is nonnegative. For convenience, we may assume that the appropriate weak solution is smooth, and no longer consider approximation problem.

Equation (1) describes the diffusion of concentration of some Newtonian fluids through porous medium or the density of some biological species in many physical phenomena and biological species theories (cf. [1-6]). It has been known that the nonlocal source term presents a more realistic model for population dynamics, see [1-3].

During the past decades, there have been many works to deal with the existence and nonexistence of global solutions, blow-up of solutions, bounds for the blow-up time, blowup rates, blow-up sets, and asymptotic behavior of the solutions to the parabolic equations. We refer the readers to the monographs [7-9] as well as the survey paper [10] and the references therein. Roughly, the existence and nonexistence of global solutions and behavior of the solutions to parabolic equations depend on space dimension, nonlinearity, initial data, and nonlinear boundary flux. Specially, Quittner and Souplet [9, Chap. 5] introduced the qualitative properties of the solution to nonlocal semilinear parabolic equation with homogeneous Dirichlet boundary condition in detail. In a sense, the nonlocal models are closer to the practical problems than the local ones, and now many local theories are no longer holding, hence, nonlocal problems are more challenging and difficult. In this paper, we would like to investigate the blow-up phenomena for the solution to a class of nonlocal problems, and our main aim is to derive the bounds for blow-up time if blow-up occurs in finite time. As far as we know, a variety of methods have been used to investigate the upper bounds for the blow-up time to the above problems (cf. [11]), while the lower bounds for the blow-up time may be harder to be determined and fewer methods can be chosen to deal with them. Recently, the study on the lower bounds for blow-up time has made some progress, while more attention was paid to the local model with constant coefficients. We provide the readers with the literature [12-14] for a three-dimensional case and [15] for a high-dimensional case.

For some research on the nonlocal reaction-diffusion equations with constant coefficients, Song [16] considered the semilinear parabolic equation with nonlocal source and local absorption

$$
u_{t}=\Delta u+\int_{\Omega} u^{q} d x-k u^{s}, \quad(x, t) \in \Omega \times\left(0, t^{*}\right)
$$

under homogeneous Dirichlet or Neumann boundary condition, and obtained the bounds for blow-up time of the solution to the initial boundary value problem in a threedimensional space. Later, Liu [17] considered the problem with nonlinear Neumann boundary condition and derived the lower bounds for blow-up time of the blow-up solution in a three-dimensional space. Recently, Tang et al. [18] generalized the results in [16] to the case of a high-dimensional space. Liu et al. [19] studied the porous medium equation with nonlocal source term

$$
u_{t}=\Delta u^{m}+\int_{\Omega} u^{q} d x, \quad(x, t) \in \Omega \times\left(0, t^{*}\right)
$$

under homogeneous Dirichlet or Neumann boundary condition. They obtained the lower bounds for blow-up time of the blow-up solution to the initial boundary value problem in 
a three-dimensional space. Specially, Fang et al. [20] studied

$$
u_{t}=\Delta u^{m}+u^{p} \int_{\Omega} u^{q} d x-u^{s}, \quad(x, t) \in \Omega \times\left(0, t^{*}\right)
$$

under homogeneous Dirichlet or Neumann boundary condition and obtained the lower bounds for blow-up time of the blow-up solution in a three-dimensional space. Bao and Song [21] considered the initial boundary value problem of quasilinear parabolic equation under homogeneous Dirichlet or Neumann boundary condition, and the slow diffusion case with nonlocal source term was also included in their results. Besides, one can refer to [22-27] for the results about scalar equation with time-dependent coefficients, nonlocal reaction systems, and models of quasilinear equations.

For some research on the nonlocal reaction-diffusion equations with weight functions, Song and Lv [28, Sect. 5] studied the semilinear parabolic equation with weighted inner source and absorption

$$
u_{t}=\Delta u+a(x) \int_{\Omega} u^{q} d x-u^{s}, \quad(x, t) \in \Omega \times\left(0, t^{*}\right)
$$

where the weight function $a(x) \in C^{1}(\Omega) \cap C^{0}(\bar{\Omega})$ satisfies $\left(a_{1}\right)$ or $\left(a_{2}\right)$. They considered the problem under homogeneous Dirichlet or Neumann boundary condition and obtained the estimate for the blow-up rate and bounds for the blow-up time of solution to the initial boundary value problem in a high-dimensional space $(N \geq 3)$. However, their results did not include the influence of weight functions on the blow-up phenomenon. Besides, one can refer to [29-31] for more results about reaction-diffusion models with spacedependent coefficients.

In view of the works mentioned above, there are few results about bounds for the blowup time of blow-up solution to the initial boundary value problem (1)-(3). The main difficulties are to seek the competitive relationship among nonlinear diffusion term, source term, and absorption, as well as to investigate the influence of space dimension and weight functions on the blow-up solution. Motivated by these observations, using a modified differential inequality technique, we can establish sufficient conditions for the blow-up of solution to problem (1)-(3) under appropriate measure and obtain the upper bounds for the blow-up time. Meanwhile, we can also derive the lower bounds for the blow-up time of blow-up solution in the whole-dimensional space $(N \geq 1)$. Indeed, for the case $p+q \leq \max \{m, s\}$, we can easily construct the global supersolution for the solution to problem (1)-(3), so we only consider the case $p+q>\max \{m, s\}$ in our paper.

Our paper is organized as follows. In Sect. 2, we assume some conditions on the weight function $a(x)$ to guarantee that the solution to problem (1)-(3) blows up in finite time under appropriate measure, and derive the upper bounds for blow-up time. In Sect. 3, we obtain the lower bounds for blow-up time of the solution to problem (1)-(3) in the whole-dimensional space.

\section{Upper bounds for the blow-up time}

In this section, we establish some sufficient conditions for the solution to problem (1)-(3) to blow up in finite time under different measures, and then derive the upper bounds for the blow-up time. 
We firstly give a sufficient condition for the solution to problem (1)-(3) to blow up in $L^{1}$-norm and establish an upper bound for the blow-up time.

Theorem 1 Suppose that $m>1, \min \{p, q\}>s$, and the weight function $a(x)$ satisfies $\left(a_{1}\right)$. If $u$ is the nonnegative solution to problem (1)-(3), then $u$ blows up in $L^{1}$-norm, and an upper bound for $t^{*}$ is

$$
\int_{J_{1}(0)}^{\infty} \frac{d \eta}{M_{1} \eta^{p+q}-M_{2}}
$$

where $J_{1}(0)=\int_{\Omega} u_{0}(x) d x$, the initial data $u_{0}(x)$ is large enough and positive constants $M_{1}$, $M_{2}$ will be given in the proof.

Proof Define

$$
J_{1}(t)=\int_{\Omega} u d x
$$

Compute the derivative and use Green's formula and Hölder's inequality to derive

$$
\begin{aligned}
J_{1}^{\prime}(t) & =\int_{\Omega} u_{t} d x \\
& =\int_{\Omega} \Delta u^{m} d x+\int_{\Omega} a(x) u^{p} d x \int_{\Omega} u^{q} d x-\int_{\Omega} u^{s} d x \\
& \geq a|\Omega|^{\frac{2 s-(p+q)}{s}}\left(\int_{\Omega} u^{s} d x\right)^{\frac{p+q}{s}}-\int_{\Omega} u^{s} d x \\
& =\int_{\Omega} u^{s} d x\left[a|\Omega|^{\frac{2 s-(p+q)}{s}}\left(\int_{\Omega} u^{s} d x\right)^{\frac{p+q-s}{s}}-1\right] .
\end{aligned}
$$

Obviously, since $p+q>s$, we can get that the function $f(\eta)=\eta^{\frac{p+q-s}{s}}$ is monotone increasing and if $u_{0}(x)$ satisfies

$$
a|\Omega|^{\frac{2 s-(p+q)}{s}}\left(\int_{\Omega} u_{0}^{s} d x\right)^{\frac{p+q-s}{s}}>1,
$$

then we can know that the solution to problem (1)-(3) blows up in finite time.

On the other hand, by (4) and Young's and Hölder's inequalities, we can derive

$$
\begin{aligned}
J_{1}^{\prime}(t) & \geq a|\Omega|^{\frac{2 s-(p+q)}{s}}\left(\int_{\Omega} u^{s} d x\right)^{\frac{p+q}{s}}-\int_{\Omega} u^{s} d x \\
& =a|\Omega|^{\frac{2 s-(p+q)}{s}}\left(\int_{\Omega} u^{s} d x\right)^{\frac{p+q}{s}}-\left(\delta_{1}\left(\int_{\Omega} u^{s} d x\right)^{\frac{p+q}{s}}\right)^{\frac{s}{p+q}}\left(\delta_{1}^{-\frac{s}{p+q-s}}\right)^{\frac{p+q-s}{p+q}} \\
& \geq\left[a|\Omega|^{\frac{2 s-(p+q)}{s}}-\frac{s \delta_{1}}{p+q}\right]\left(\int_{\Omega} u^{s} d x\right)^{\frac{p+q}{s}}-\frac{p+q-s}{p+q} \delta_{1}^{-\frac{s}{p+q-s}} \\
& \geq\left[a|\Omega|^{\frac{2 s-(p+q)}{s}}-\frac{s \delta_{1}}{p+q}\right]|\Omega|^{\frac{(1-s)(p+q)}{s}}\left(\int_{\Omega} u d x\right)^{p+q}-\frac{p+q-s}{p+q} \delta_{1}^{-\frac{s}{p+q-s}} .
\end{aligned}
$$


Choosing $\delta_{1}$ satisfies $0<\delta_{1}<\frac{a(p+q)}{s}|\Omega|^{\frac{2 s-(p+q)}{s}}$, then (6) can be rewritten as

$$
J_{1}^{\prime}(t) \geq M_{1} J_{1}^{p+q}-M_{2}
$$

where

$$
M_{1}=\left(a|\Omega|^{\frac{2 s-(p+q)}{s}}-\frac{s \delta_{1}}{p+q}\right)|\Omega|^{\frac{(1-s)(p+q)}{s}}>0, \quad M_{2}=\frac{p+q-s}{p+q} \delta_{1}^{-\frac{s}{p+q-s}}>0 .
$$

Hence, if $u_{0}(x)$ is large enough satisfying $J_{1}(0)=\int_{\Omega} u_{0}(x) d x>\left(\frac{M_{2}}{M_{1}}\right)^{\frac{1}{p+q}}$ and (5), by virtue of (7), we can derive that the blow-up time $t^{*}$ satisfies

$$
t^{*} \leq \int_{J_{1}(0)}^{\infty} \frac{d \eta}{M_{1} \eta^{p+q}-M_{2}}
$$

Next we will use Kaplan's method to investigate the upper bound for blow-up time of blow-up solution to problem (1)-(3).

Denote by $\lambda_{1}$ and $\phi_{1}$ the first eigenvalue and the corresponding eigenfunction of the following fixed membrane problem:

$$
\begin{aligned}
& \Delta \phi_{1}+\lambda_{1} \phi_{1}=0, \quad x \in \Omega, \\
& \phi_{1}(x)=0, \quad x \in \partial \Omega .
\end{aligned}
$$

It is well known that $\phi_{1}$ may be normalized as $\sup _{\Omega}\left|\phi_{1}\right|=1$.

Theorem 2 Suppose that $1 \leq m<s<\min \{p, q\}$, and the weight function $a(x)$ satisfies $\left(a_{1}\right)$. Define an auxiliary function

$$
J_{2}(t)=\int_{\Omega} u \phi_{1} d x
$$

If $u$ is the nonnegative solution to problem (1)-(3), then $u$ blows up in the measure of $J_{2}$, and an upper bound for $t^{*}$ is

$$
\int_{J_{2}(0)}^{\infty} \frac{d \eta}{M_{3} \eta^{p+q}-M_{4}}
$$

where $J_{2}(0)=\int_{\Omega} u_{0}(x) \phi_{1}(x) d x$, the initial data $u_{0}(x)$ is large enough and positive constants $M_{3}, M_{4}$ will be given in the proof.

Proof We compute the derivative and use Green's formula to obtain

$$
\begin{aligned}
J_{2}^{\prime}(t) & =\int_{\Omega} u_{t} \phi_{1} d x \\
& =\int_{\Omega} \Delta u^{m} \phi_{1} d x+\int_{\Omega} a(x) u^{p} \phi_{1} d x \int_{\Omega} u^{q} d x-\int_{\Omega} u^{s} \phi_{1} d x \\
& \geq-\lambda_{1} \int_{\Omega} u^{m} \phi_{1} d x+a \int_{\Omega} u^{p} \phi_{1} d x \int_{\Omega} u^{q} \phi_{1} d x-\int_{\Omega} u^{s} \phi_{1} d x
\end{aligned}
$$


Notice that $m<s<\min \{p, q\}$, by Hölder's inequality to estimate the first and second terms on the right-hand side of (10), we have

$$
\begin{aligned}
\int_{\Omega} u^{m} \phi_{1} d x \leq\left(\int_{\Omega} u^{s} \phi_{1} d x\right)^{\frac{m}{s}}\left(\int_{\Omega} \phi_{1} d x\right)^{\frac{s-m}{s}} \leq\left(\int_{\Omega} u^{s} \phi_{1} d x\right)^{\frac{m}{s}}|\Omega|^{\frac{s-m}{s}}, \\
\int_{\Omega} u^{p} \phi_{1} d x \int_{\Omega} u^{q} \phi_{1} d x \geq\left(\int_{\Omega} u^{s} \phi_{1} d x\right)^{\frac{p+q}{s}}\left(\int_{\Omega} \phi_{1} d x\right)^{\frac{2 s-(p+q)}{s}} \\
\geq\left(\int_{\Omega} u^{s} \phi_{1} d x\right)^{\frac{p+q}{s}}|\Omega|^{\frac{2 s-(p+q)}{s}} .
\end{aligned}
$$

Substituting (11) into (10) and using Young's inequality, we obtain

$$
\begin{aligned}
J_{2}^{\prime}(t) \geq & a|\Omega|^{\frac{2 s-(p+q)}{s}}\left(\int_{\Omega} u^{s} \phi_{1} d x\right)^{\frac{p+q}{s}}-\lambda_{1}|\Omega|^{\frac{s-m}{s}}\left(\int_{\Omega} u^{s} \phi_{1} d x\right)^{\frac{m}{s}} \\
& -\int_{\Omega} u^{s} \phi_{1} d x \\
\geq & a|\Omega|^{\frac{2 s-(p+q)}{s}}\left(\int_{\Omega} u^{s} \phi_{1} d x\right)^{\frac{p+q}{s}}-\left(\frac{m}{s} \lambda_{1}^{\frac{s}{m}}+1\right) \int_{\Omega} u^{s} \phi_{1} d x-\frac{s-m}{s}|\Omega| .
\end{aligned}
$$

By the property of quadratic function and $a>0$, if $u_{0}(x)$ is large enough, we can derive that the solution to problem (1)-(3) blows up in finite time.

Now, applying the same argument in the proof of Theorem 2 to (12), we obtain

$$
J_{2}^{\prime}(t) \geq\left(a|\Omega|^{\frac{2 s-(p+q)}{s}}-\frac{m \delta_{2}+s \delta_{3}}{p+q}\right)\left(\int_{\Omega} u^{s} \phi_{1} d x\right)^{\frac{p+q}{s}}-M_{4} .
$$

Choosing $\delta_{2}, \delta_{3}$ satisfies $0<\delta_{2}<\frac{(p+q) a|\Omega|^{2 s-(p+q)}}{2 m}$ and $0<\delta_{3}<\frac{(p+q) a|\Omega|^{2 s-(p+q)}}{2 s}$, using Hölder's inequality, (13) can be rewritten as

$$
J_{2}^{\prime}(t) \geq M_{3} J_{2}^{p+q}-M_{4}
$$

where

$$
\begin{aligned}
& M_{3}=\left(a|\Omega|^{\frac{2 s-(p+q)}{s}}-\frac{m \delta_{2}+s \delta_{3}}{p+q}\right)|\Omega|^{\frac{(1-s)(p+q)}{s}}>0, \\
& M_{4}=\frac{p+q-m}{p+q} \delta_{2}^{-\frac{m}{p+q-m}} \lambda_{1}^{\frac{p+q}{p+q}}|\Omega|^{\frac{(s-m)(p+q)}{s(p+q-m)}}+\frac{p+q-s}{p+q} \delta_{3}^{-\frac{s}{p+q-s}}>0 .
\end{aligned}
$$

Hence, if $u_{0}(x)$ is large enough and satisfies $J_{2}(0)=\int_{\Omega} u_{0}(x) \phi_{1}(x) d x>\left(\frac{M_{4}}{M_{3}}\right)^{\frac{1}{p+q}}$ and the above requirement, by (14), we obtain

$$
t^{*} \leq \int_{J_{2}(0)}^{\infty} \frac{d \eta}{M_{3} \eta^{p+q}-M_{4}} .
$$

Afterwards, we will seek a sufficient condition for the solution to problem (1)-(3) to blow up in $L^{2 m}$-norm, and then obtain an upper bound for the blow-up time. 
Theorem 3 Suppose that $m>1, p \geq s>1$, and the weight function $a(x)$ satisfies $\left(a_{1}\right)$ or $\left(a_{2}\right)$. Define auxiliary functions

$$
\begin{aligned}
& F\left(u^{m}\right)=\int_{0}^{u^{m}} \xi^{1+\frac{p-1}{m}}\left(\int_{\Omega} \xi^{\frac{q}{m}} d x\right) d \xi \\
& G\left(u^{m}\right)=\int_{0}^{u^{m}} \xi^{1+\frac{s-1}{m}} d \xi=\frac{m}{2 m+s-1} u^{2 m+s-1} .
\end{aligned}
$$

Assume that there exist $I_{1} \geq I_{2}>2$ such that

$$
u^{2 m+p-1} \int_{\Omega} u^{q} d x \geq I_{1} F\left(u^{m}\right), \quad u^{2 m+s-1} \leq I_{2} G\left(u^{m}\right) .
$$

If $u$ is the nonnegative solution to problem (1)-(3) and the initial data $u_{0}(x)$ satisfies

$$
-\frac{1}{2} \int_{\Omega} \nabla u_{0}^{2 m-1} \nabla u_{0}^{m} d x+\int_{\Omega} a(x) F\left(u_{0}^{m}\right) d x-\int_{\Omega} G\left(u_{0}^{m}\right) d x>0,
$$

then $u$ blows up in $L^{2 m}$-norm, and an upper bound for $t^{*}$ is

$$
\frac{2 \xi(0)}{I_{2}\left(I_{2}-2\right) \eta(0)}
$$

where functions $\xi, \eta$ will be given in the proof.

Remark 1 Since $p \geq s>1$, we can choose $I_{1}=\frac{2 m+p-1}{m}, I_{2}=\frac{2 m+s-1}{m}$, which satisfy condition (15).

Proof Applying the transformation $v=u^{m}$ in (1), we have

$$
\left(v^{\frac{1}{m}}\right)_{t}=\Delta v+a(x) v^{\frac{p}{m}} \int_{\Omega} v^{\frac{q}{m}} d x-v^{\frac{s}{m}}
$$

Then (16) is equivalent to

$$
v_{t}=m v^{1-\frac{1}{m}}\left(\Delta v+a(x) v^{\frac{p}{m}} \int_{\Omega} v^{\frac{q}{m}} d x-v^{\frac{s}{m}}\right) .
$$

Define

$$
\xi(t)=\int_{\Omega} u^{2 m} d x=\int_{\Omega} v^{2} d x .
$$

Differentiating $\xi(t)$ and using (15), (17) and Green's formula, we obtain

$$
\begin{aligned}
\xi^{\prime}(t)= & 2 \int_{\Omega} v v_{t} d x \\
= & -2 m \int_{\Omega} \nabla v^{2-\frac{1}{m}} \cdot \nabla v d x+2 m \int_{\Omega} a(x) v^{2+\frac{p-1}{m}} d x \int_{\Omega} v^{\frac{q}{m}} d x \\
& -2 m \int_{\Omega} v^{2+\frac{s-1}{m}} d x
\end{aligned}
$$




$$
\begin{aligned}
& \geq-I_{2} m \int_{\Omega} \nabla v^{2-\frac{1}{m}} \cdot \nabla v d x+2 I_{1} m \int_{\Omega} a(x) F(v) d x-2 I_{2} m \int_{\Omega} G(v) d x \\
& \geq 2 I_{2} \eta(t)
\end{aligned}
$$

where $\eta(t):=-\frac{m}{2} \int_{\Omega} \nabla v^{2-\frac{1}{m}} \cdot \nabla v d x+m \int_{\Omega} a(x) F(v) d x-m \int_{\Omega} G(v) d x$.

Next, differentiating $\eta(t)$ and using Green's formula and $m>1$, we have

$$
\begin{aligned}
\eta^{\prime}(t)= & -\frac{m}{2} \int_{\Omega} \nabla\left(v^{2-\frac{1}{m}}\right)_{t} \cdot \nabla v d x-\frac{m}{2} \int_{\Omega} \nabla v^{2-\frac{1}{m}} \cdot \nabla v_{t} d x \\
& +m \int_{\Omega} a(x) v^{1+\frac{p-1}{m}}\left(\int_{\Omega} v^{\frac{q}{m}} d x\right) v_{t} d x-m \int_{\Omega} v^{1+\frac{s-1}{m}} v_{t} d x \\
= & m\left(2-\frac{1}{m}\right) \int_{\Omega} v^{1-\frac{1}{m}} v_{t} \Delta v d x \\
& +\frac{m}{2}\left(2-\frac{1}{m}\right)\left(1-\frac{1}{m}\right) \int_{\Omega} v^{-\frac{1}{m}}|\nabla v|^{2} v_{t} d x \\
& +m \int_{\Omega} a(x) v^{1+\frac{p-1}{m}}\left(\int_{\Omega} v^{\frac{q}{m}} d x\right) v_{t} d x-m \int_{\Omega} v^{1+\frac{s-1}{m}} v_{t} d x \\
\geq & m \int_{\Omega} v^{1-\frac{1}{m}} \Delta v \cdot v_{t} d x+m \int_{\Omega} a(x) v^{1+\frac{p-1}{m}}\left(\int_{\Omega} v^{\frac{q}{m}} d x\right) v_{t} d x \\
& -m \int_{\Omega} v^{1+\frac{s-1}{m}} v_{t} d x \\
= & \int_{\Omega}\left(v_{t}\right)^{2} d x \geq 0 .
\end{aligned}
$$

By the hypotheses in Theorem 3 , we can easily see that $\eta(0)>0$, and then $\eta(t)>0, \forall t \geq 0$. Therefore, (19) implies that $\xi$ is monotone increasing, and then $v$ blows up in finite time $t^{*}$ in $L^{2}$-norm.

Combining (18)-(20) and using Schwarz's inequality

$$
\left(\int_{\Omega} v v_{t} d x\right)^{2} \leq \int_{\Omega} v^{2} d x \int_{\Omega}\left(v_{t}\right)^{2} d x
$$

we can derive

$$
4 \xi(t) \eta^{\prime}(t) \geq\left(\xi^{\prime}(t)\right)^{2} \geq 2 I_{2} \xi^{\prime}(t) \eta(t) .
$$

Applying (19) and (21), we can compute

$$
\eta(t) \geq \frac{\eta(0)}{(\xi(0))^{\frac{I_{2}}{2}}}(\xi(t))^{\frac{I_{2}}{2}}
$$

and

$$
\xi^{\prime}(t) \geq \frac{2 I_{2} \eta(0)}{(\xi(0))^{\frac{I_{2}}{2}}}(\xi(t))^{\frac{I_{2}}{2}} .
$$

Hence, (22) leads to

$$
t^{*} \leq \frac{\xi(0)}{I_{2}\left(I_{2}-2\right) \eta(0)}
$$


Remark 2 In the fast and linear diffusion situation $(0<m \leq 1)$, we set

$$
F(u)=\int_{0}^{u} \xi^{p}\left(\int_{\Omega} \xi^{q} d x\right) d \xi, \quad G(u)=\int_{0}^{u} \xi^{s} d s=\frac{1}{s+1} u^{s+1} .
$$

Suppose that there exist $I_{3} \geq I_{4}>2$ such that

$$
u^{p+1} \int_{\Omega} u^{q} d x \geq I_{3} F(u), \quad u^{s+1} \leq I_{4} G(u) .
$$

If $u$ is a nonnegative solution to problem (1)-(3) and the initial data $u_{0}(x)$ satisfies

$$
-\frac{1}{2} \int_{\Omega} \nabla u_{0} \nabla u_{0}^{m} d x+\int_{\Omega} a(x) F\left(u_{0}\right) d x-\int_{\Omega} G\left(u_{0}\right) d x>0,
$$

then $u$ blows up in $L^{2}$-norm, and an upper bound for $t^{*}$ is

$$
t^{*} \leq \frac{\chi(0)}{I_{4}\left(I_{4}-2\right) \zeta(0)}
$$

where $\chi(t)=\int_{\Omega} u^{2} d x, \zeta(t)=-\frac{1}{2} \int_{\Omega} \nabla u \nabla u^{m} d x+\int_{\Omega} a(x) F(u) d x-\int_{\Omega} G(u) d x$.

The proof is the same as Theorem 3 , so we omit it.

\section{Lower bounds for the blow-up time}

In this section, we seek the lower bounds for the blow-up time of the solution to problem (1)-(3) in an $N$-dimensional space $\Omega \subset \mathbf{R}^{N}(N \geq 1)$.

\section{1 $N=1$ case}

Suppose $\Omega=(0, l), N=1$, then problem (1)-(3) can be rewritten as

$$
\begin{aligned}
& u_{t}=\left(u^{m}\right)_{x x}+a(x) u^{p} \int_{0}^{l} u^{q} d x-u^{s}, \quad x \in(0, l), t \in\left(0, t^{*}\right), \\
& u(0, t)=u(l, t)=0, \quad t \in\left(0, t^{*}\right) \\
& u(x, 0)=u_{0}(x) \geq 0, \quad x \in(0, l) .
\end{aligned}
$$

Theorem 4 Suppose that $m>0,0 \leq p<1, q>1, \Omega=(0, l)$ and the weight function $a(x)$ satisfies $\left(a_{1}\right)$ or $\left(a_{2}\right)$. Define an auxiliary function

$$
\psi_{1}(t)=\int_{0}^{l} u^{k+1} d x
$$

where $k>\max \{2-m, q-1\}$. If the solution $u$ to problem (23)-(25) blows up in $L^{k+1}$-norm at $t^{*}$, then $t^{*}$ is bounded below by

$$
\int_{\psi_{1}(0)}^{\infty} \frac{d \eta}{H_{1} \eta^{\frac{k+p+q}{k+1}}}
$$

where $\psi_{1}(0)=\int_{0}^{l} u_{0}^{k+1}(x) d x$, positive constant $H_{1}$ will be given in the proof. 
Proof Differentiating $\psi_{1}(t)$ and using Green's formula and Hölder's inequality, we have

$$
\begin{aligned}
\psi_{1}^{\prime}(t)= & (k+1) \int_{0}^{l} u^{k} u_{t} d x \\
= & -m k(k+1) \int_{0}^{l} u^{k+m-2} u_{x}^{2} d x+(k+1) \int_{0}^{l} a(x) u^{k+p} d x \int_{0}^{l} u^{q} d x \\
& -(k+1) \int_{0}^{l} u^{k+s} d x \\
\leq & H_{1}\left(\int_{0}^{l} u^{k+1} d x\right)^{\frac{k+p+q}{k+1}},
\end{aligned}
$$

where $H_{1}=(k+1) l^{\frac{k+1-q}{k+1}}\left(\int_{0}^{l}(a(x))^{\frac{k+1}{1-p}} d x\right)^{\frac{1-p}{k+1}}$.

Hence, applying (26), we can derive that the lower bound for $t^{*}$ satisfies

$$
t^{*} \geq \int_{\psi_{1}(0)}^{\infty} \frac{d \eta}{H_{1} \eta^{\frac{k+p+q}{k+1}}}
$$

\section{$3.2 N=2$ case}

Theorem 5 Suppose that $m>0,0 \leq p<1, q>1, \Omega \subset \mathbf{R}^{2}$ and the weight function satisfies $\left(a_{2}\right)$ and

$\left(a_{3}\right)$ there exists $A=\left(A_{1}, A_{2}\right)$ such that $-a(x) A \leq \nabla a(x) \leq a(x) A$,

where $x \in \Omega, A_{i}>0, i=1,2$, while A satisfies $|A|^{2}<\lambda_{1}$. Define a weight function

$$
\psi_{2}(t)=\int_{\Omega} a(x) u^{k+1} d x
$$

where $\lambda_{1}$ is the first eigenvalue of the fixed membrane problem (8)-(9) for a two-dimensional space, $k>\max \left\{q-1, \frac{|A| m}{2\left(\sqrt{\lambda_{1}}-|A|\right)}\right\}$. If the solution $u$ to problem $(1)-(3)$ blows up in the measure $\psi_{2}$ at $t^{*}$, then $t^{*}$ is bounded below by

$$
\int_{\psi_{2}(0)}^{\infty} \frac{d \eta}{H_{2} \eta^{\frac{k+p+q}{k+1}}}
$$

where $\psi_{2}(0)=\int_{\Omega} a(x) u_{0}^{k+1}(x) d x$, positive constant $H_{2}$ will be given in the proof.

In order to prove Theorem 5, we firstly need to give a lemma.

Lemma 1 Suppose that a $(x)$ satisfies $\left(a_{2}\right),\left(a_{3}\right)$, and $|A|^{2}<\lambda_{1}$. If $u \in C^{1}(\Omega)$ is nonnegative, then we have the differential inequality

$$
\left(\sqrt{\lambda_{1}}-\frac{|A|}{2}\right)\left(\int_{\Omega} a(x) u^{2 k} d x\right)^{\frac{1}{2}} \leq\left(\int_{\Omega} a(x)\left|\nabla u^{k}\right|^{2} d x\right)^{\frac{1}{2}}, \quad k>0, x \in \Omega
$$

Proof By virtue of the Rayleigh principle, we know

$$
\lambda_{1} \int_{\Omega} \omega^{2} d x \leq \int_{\Omega}|\nabla \omega|^{2} d x, \quad \text { where }\left.\omega\right|_{\partial \Omega}=0
$$


Now, choosing $\omega=a^{\frac{1}{2}}(x) u^{k}$, by $\left(a_{3}\right)$ and Hölder's inequality, we have

$$
\begin{aligned}
& \lambda_{1} \int_{\Omega} a(x) u^{2 k} d x \\
& \leq \int_{\Omega}\left|\nabla\left(a^{\frac{1}{2}}(x) u^{k}\right)\right|^{2} d x \\
&=\frac{1}{4} \int_{\Omega} \frac{|\nabla a(x)|^{2}}{a(x)} u^{2 k} d x+\int_{\Omega} a(x)\left|\nabla u^{k}\right|^{2} d x+\int_{\Omega} u^{k} \nabla a(x) \nabla u^{k} d x \\
& \leq \frac{|A|^{2}}{4} \int_{\Omega} a(x) u^{2 k} d x+\int_{\Omega} a(x)\left|\nabla u^{k}\right|^{2} d x \\
&+|A|\left(\int_{\Omega} a(x) u^{2 k} d x\right)^{\frac{1}{2}}\left(\int_{\Omega} a(x)\left|\nabla u^{k}\right|^{2} d x\right)^{\frac{1}{2}} \\
& \leq {\left[\frac{|A|}{2}\left(\int_{\Omega} a(x) u^{2 k} d x\right)^{\frac{1}{2}}+\left(\int_{\Omega} a(x)\left|\nabla u^{k}\right|^{2} d x\right)^{\frac{1}{2}}\right]^{2} . }
\end{aligned}
$$

Hence, we derive

$$
\left(\sqrt{\lambda_{1}}-\frac{|A|}{2}\right)\left(\int_{\Omega} a(x) u^{2 k} d x\right)^{\frac{1}{2}} \leq\left(\int_{\Omega} a(x)\left|\nabla u^{k}\right|^{2} d x\right)^{\frac{1}{2}} .
$$

The proof of Theorem 5 can be given as follows.

Proof Differentiating $\psi_{2}(t)$ and using Green's formula, Hölder's inequality, and Lemma 1, we have

$$
\begin{aligned}
\psi_{2}^{\prime}(t)= & (k+1) \int_{\Omega} a(x) u^{k} u_{t} d x \\
= & -(k+1) \int_{\Omega}\left(\nabla a(x) u^{k}+k a(x) u^{k-1} \nabla u\right) m u^{m-1} \nabla u d x \\
& +(k+1) \int_{\Omega} a^{2}(x) u^{k+p} d x \int_{\Omega} u^{q} d x-(k+1) \int_{\Omega} a(x) u^{k+s} d x \\
\leq & \frac{2 m(k+1)|A|}{k+m} \int_{\Omega} a(x) u^{\frac{k+m}{2}}\left|\nabla u^{\frac{k+m}{2}}\right| d x \\
& -\frac{4 m k(k+1)}{(k+m)^{2}} \int_{\Omega} a(x)\left|\nabla u^{\frac{k+m}{2}}\right|^{2} d x \\
& +(k+1) \int_{\Omega} a^{2}(x) u^{k+p} d x \int_{\Omega} u^{q} d x-(k+1) \int_{\Omega} a(x) u^{k+s} d x \\
\leq & \frac{2 m(k+1)|A|}{k+m}\left(\int_{\Omega} a(x) u^{k+m} d x\right)^{\frac{1}{2}}\left(\int_{\Omega} a(x)\left|\nabla u^{\frac{k+m}{2}}\right|^{2} d x\right)^{\frac{1}{2}} \\
& -\frac{4 m k(k+1)}{(k+m)^{2}} \int_{\Omega} a(x)\left|\nabla u^{\frac{k+m}{2}}\right|^{2} d x \\
& +(k+1) \int_{\Omega} a^{2}(x) u^{k+p} d x \int_{\Omega} u^{q} d x \\
\leq & {\left[\frac{4 m(k+1)|A|}{\left(2 \sqrt{\lambda_{1}}-|A|\right)(k+m)}-\frac{4 m k(k+1)}{(k+m)^{2}}\right] \int_{\Omega} a(x)\left|\nabla u^{\frac{k+m}{2}}\right|^{2} d x } \\
& +(k+1) \int_{\Omega} a^{2}(x) u^{k+p} d x \int_{\Omega} u^{q} d x .
\end{aligned}
$$


By the value of $k$, we can easily get that the coefficient of $\int_{\Omega} a(x)\left|\nabla u^{\frac{k+m}{2}}\right|^{2} d x$ in (28) is negative. Then, applying Hölder's inequality, we have

$$
\psi_{2}^{\prime}(t) \leq H_{2} \psi_{2}^{\frac{k+p+q}{k+1}}
$$

where $H_{2}=(k+1)\left(\int_{\Omega}(a(x))^{\frac{k+2-p}{1-p}} d x\right)^{\frac{1-p}{k+1}}\left(\int_{\Omega}(a(x))^{-\frac{q}{k+1-q}} d x\right)^{\frac{k+1-q}{k+1}}$.

Hence, using (27), we can obtain that the lower bound for $t^{*}$ satisfies

$$
t^{*} \geq \int_{\psi_{2}(0)}^{\infty} \frac{d \eta}{H_{2} \eta^{\frac{k+p+q}{k+1}}} .
$$

\section{3 $N \geq 3$ case}

Theorem 6 Suppose that $m>0, p+q>\max \{m, s\}, \Omega \subset \mathbf{R}^{N}(N \geq 3)$ is a bounded convex region with smooth boundary, the weight function $a(x)$ satisfies $\left(a_{1}\right)$ or $\left(a_{2}\right)$ and

$\left(a_{3}\right)^{\prime}$ there exists $A=\left(A_{1}, \ldots, A_{N}\right)$ such that $-a(x) A \leq \nabla a(x) \leq a(x) A$,

where $x \in \Omega, A_{i}>0, i=1, \ldots, N, N \geq 3$.

Define a weight function

$$
\psi_{3}(t)=\int_{\Omega} a(x) u^{N k} d x
$$

where $\lambda_{1}$ is the first eigenvalue of the fixed membrane problem (8)-(9) for the Ndimensional space, $k>\max \left\{\frac{1}{N}, \frac{2(N-2)(p+q-1)}{N}, \frac{2-2 m}{3}\right\}$. If the solution $u$ to problem (1)-(3) blows up in the measure $\psi_{3}$ at $t^{*}$, then $t^{*}$ is bounded below by

$$
\int_{\psi_{3}(0)}^{\infty} \frac{d \eta}{H_{3} \eta^{\frac{(N-2) \theta}{N-2-N(1-\theta) \gamma}}+H_{4} \eta^{\frac{(N-2) \theta}{N-2-N(1-\theta)}+H_{5}}},
$$

where $\psi_{3}(0)=\int_{\Omega} a(x) u_{0}^{N k}(x) d x$ and positive constants $H_{3}, H_{4}, H_{5}, \gamma, \theta$ will be given in the proof.

Proof Differentiating $\psi_{3}(t)$ and using Green's formula, Hölder's, and Young's inequalities, and condition $\left(a_{3}\right)^{\prime}$, we have

$$
\begin{aligned}
\psi_{3}^{\prime}(t)= & N k \int_{\Omega} a(x) u^{N k-1} u_{t} d x \\
= & N k \int_{\Omega} a(x) u^{N k-1} \Delta u^{m} d x+N k \int_{\Omega} a^{2}(x) u^{N k+p-1} d x \int_{\Omega} u^{q} d x \\
& -N k \int_{\Omega} a(x) u^{N k+s-1} d x \\
= & -m N k(N k-1) \int_{\Omega} a(x) u^{N k+m-3}|\nabla u|^{2} d x \\
& -m N k \int_{\Omega} \nabla a(x) u^{N k+m-2} \nabla u d x \\
& +N k \int_{\Omega} a^{2}(x) u^{N k+p-1} d x \int_{\Omega} u^{q} d x-N k \int_{\Omega} a(x) u^{N k+s-1} d x \\
\leq & -\frac{4 m N k(N k-1)}{(N k+m-1)^{2}} \int_{\Omega} a(x)\left|\nabla u^{\frac{N k+m-1}{2}}\right|^{2} d x
\end{aligned}
$$




$$
\begin{aligned}
& +m N k|A|\left(\int_{\Omega} a(x) u^{N k+m-1} d x\right)^{\frac{1}{2}} \\
& \times\left(\frac{4}{(N k+m-1)^{2}} \int_{\Omega} a(x)\left|\nabla u^{\frac{N k+m-1}{2}}\right|^{2} d x\right)^{\frac{1}{2}} \\
& +N k \int_{\Omega} a^{2}(x) u^{N k+p-1} d x \int_{\Omega} u^{q} d x-N k \int_{\Omega} a(x) u^{N k+s-1} d x \\
& \leq \\
& {\left[-\frac{4 m N k(N k-1)}{(N k+m-1)^{2}}+\frac{2 m N k|A| \varepsilon_{1}}{(N k+m-1)^{2}}\right] \int_{\Omega} a(x)\left|\nabla u^{\frac{N k+m-1}{2}}\right|^{2} d x} \\
& +\frac{m N k|A|}{2 \varepsilon_{1}} \int_{\Omega} a(x) u^{N k+m-1} d x+N k \int_{\Omega} a^{2}(x) u^{N k+p-1} d x \int_{\Omega} u^{q} d x \\
& -N k \int_{\Omega} a(x) u^{N k+s-1} d x,
\end{aligned}
$$

where $\varepsilon_{1}$ is a positive constant to be determined later.

To begin with, applying Hölder's inequality, we get the inequality

$$
\begin{aligned}
& \int_{\Omega} a^{\frac{N-2 \theta}{N-2}}(x) u^{\frac{N k(2 N-3)}{2(N-2)}} d x \\
& \quad \leq\left(\int_{\Omega} a(x) u^{N k} d x\right)^{\theta}\left(\int_{\Omega}\left(a^{\frac{1}{2}}(x) u^{\frac{N k+m-1}{2}}\right)^{\frac{2 N}{N-2}} d x\right)^{1-\theta},
\end{aligned}
$$

where $\theta=\frac{3 k+2 m-2}{4 k+2 m-2}$. Using Hölder's and Young's inequalities to estimate the second term on the right-hand side of (29), we have

$$
\begin{aligned}
\int_{\Omega} a(x) u^{N k+m-1} d x & \leq\left(\int_{\Omega} a^{\frac{N-2 \theta}{N-2}}(x) u^{\frac{N k(2 N-3)}{2(N-2)}} d x\right)^{\gamma}\left(\int_{\Omega}(a(x))^{\sigma_{1}} d x\right)^{1-\gamma} \\
& \leq \gamma \int_{\Omega} a^{\frac{N-2 \theta}{N-2}}(x) u^{\frac{N k(2 N-3)}{2(N-2)}} d x+(1-\gamma) \int_{\Omega}(a(x))^{\sigma_{1}} d x,
\end{aligned}
$$

where $\gamma=\frac{2(N-2)(N k+m-1)}{N k(2 N-3)}, \sigma_{1}$ such that $1=\frac{N-2 \theta}{N-2} \gamma+\sigma_{1}(1-\gamma)$.

Next, applying Hölder's inequality to the third term on the right-hand side of (29), we obtain the inequality

$$
\begin{aligned}
& \int_{\Omega} a^{2}(x) u^{N k+p-1} d x \int_{\Omega} u^{q} d x \\
& \leq\left(\int_{\Omega}(a(x))^{\sigma_{2}} d x\right)^{\frac{q}{N k+p+q-1}}\left(\int_{\Omega}(a(x))^{\sigma_{3}} d x\right)^{\frac{N k+p-1}{N k+p+q-1}} \\
& \quad \times \int_{\Omega} a^{m_{1}+\frac{N-2 \theta}{N-2} m_{2}}(x) u^{N k+p+q-1} d x,
\end{aligned}
$$

where $m_{1}=\frac{N k-2 N(p+q)+2 N+4(p+q)-4}{N k-2 N s+2 N+4 s-4}, m_{2}=\frac{2(p+q-s)(N-2)}{N k-2 N s+2 N+4 s-4}, \sigma_{2}, \sigma_{3}$ such that

$$
\begin{aligned}
& 2=\frac{N k+p-1}{N k+p+q-1}\left(m_{1}+\frac{N-2 \theta}{N-2} m_{2}\right)+\frac{q}{N k+p+q-1} \sigma_{2}, \\
& 0=\frac{q}{N k+p+q-1}\left(m_{1}+\frac{N-2 \theta}{N-2} m_{2}\right)+\frac{N k+p-1}{N k+p+q-1} \sigma_{3} .
\end{aligned}
$$


Thus, applying Hölder's and Young's inequalities to the third integral term on the righthand side of (32), we can derive the inequality

$$
\begin{aligned}
& \int_{\Omega} a^{m_{1}+\frac{N-2 \theta}{N-2} m_{2}}(x) u^{N k+p+q-1} d x \\
& \quad \leq\left(\int_{\Omega} a(x) u^{N k+s-1} d x\right)^{m_{1}}\left(\int_{\Omega} a^{\frac{N-2 \theta}{N-2}}(x) u^{\frac{N k(2 N-3)}{2(N-2)}} d x\right)^{m_{2}} \\
& \quad \leq m_{1} \varepsilon_{2} \int_{\Omega} a(x) u^{N k+s-1} d x+m_{2} \varepsilon_{2}^{-\frac{m_{1}}{m_{2}}} \int_{\Omega} a^{\frac{N-2 \theta}{N-2}}(x) u^{\frac{N k(2 N-3)}{2(N-2)}} d x,
\end{aligned}
$$

where $\varepsilon_{2}>0$ is a constant to be determined later.

Now, we substitute (31)-(33) into (29) and choose suitable $\varepsilon_{2}>0$ to make the coefficient of $\int_{\Omega} a(x) u^{N k+s-1} d x$ in (29) vanish, that is,

$$
m_{1}\left(\int_{\Omega}(a(x))^{\sigma_{2}} d x\right)^{\frac{p}{N k+p+q-1}}\left(\int_{\Omega}(a(x))^{\sigma_{3}} d x\right)^{\frac{N k+q-1}{N k+p+q-1}} \varepsilon_{2}=1 .
$$

We can obtain the inequality

$$
\psi_{3}^{\prime}(t) \leq C_{1} \int_{\Omega} a(x)\left|\nabla u^{\frac{N k+m-1}{2}}\right|^{2} d x+C_{2} \int_{\Omega} a^{\frac{N-2 \theta}{N-2}}(x) u^{\frac{N k(2 N-3)}{2(N-2)}} d x+C_{3},
$$

where

$$
\begin{aligned}
& C_{1}=-\frac{4 m N k(N k-1)}{(N k+m-1)^{2}}+\frac{2 m N k|A| \varepsilon_{1}}{(N k+m-1)^{2}} \\
& C_{2}=N k m_{2} \varepsilon_{2} \varepsilon^{-\frac{m_{1}}{m_{2}}}\left(\int_{\Omega}(a(x))^{\sigma_{2}} d x\right)^{\frac{p}{N k+p+q-1}}\left(\int_{\Omega}(a(x))^{\sigma_{3}} d x\right)^{\frac{N k+q-1}{N k+q+q-1}}+\frac{m N k|A| \gamma}{2 \varepsilon_{1}}, \\
& C_{3}=\frac{m N k|A|(1-\gamma)}{2 \varepsilon_{1}} \int_{\Omega}(a(x))^{\sigma_{1}} d x .
\end{aligned}
$$

In order to deal with the gradient term in (34), we will use Sobolev's inequality

$$
\left(\int_{\Omega}\left(a^{\frac{1}{2}}(x) u^{\frac{N k+m-1}{2}}\right)^{\frac{2 N}{N-2}} d x\right)^{\frac{N-2}{2 N}} \leq C_{s}\left(\int_{\Omega}\left|\nabla\left(a^{\frac{1}{2}}(x) u^{\frac{N k+m-1}{2}}\right)\right|^{2} d x\right)^{\frac{1}{2}},
$$

where $C_{s}$ is the optimal Sobolev constant. Applying condition $\left(a_{3}\right)^{\prime}$ and Hölder's inequality to the right-hand side of (35), we have

$$
\begin{aligned}
& \int_{\Omega}\left|\nabla\left(a^{\frac{1}{2}}(x) u^{\frac{N k+m-1}{2}}\right)\right|^{2} d x \\
& \leq \frac{|A|^{2}}{4} \int_{\Omega} a(x) u^{N k+m-1} d x+\int_{\Omega} a(x)\left|\nabla u^{\frac{N k+m-1}{2}}\right|^{2} d x \\
& \quad+|A| \int_{\Omega} a(x) u^{\frac{N k+m-1}{2}}\left|\nabla u^{\frac{N k+m-1}{2}}\right| d x \\
& \leq {\left[\frac{|A|}{2}\left(\int_{\Omega} a(x) u^{N k+m-1} d x\right)^{\frac{1}{2}}+\left(\int_{\Omega} a(x)\left|\nabla u^{\frac{N k+m-1}{2}}\right|^{2} d x\right)^{\frac{1}{2}}\right]^{2} . }
\end{aligned}
$$


Now, using (30), (35), and (36) to estimate the second term on the right-hand side of (34), we obtain

$$
\begin{aligned}
\int_{\Omega} a^{\frac{N-2 \theta}{N-2}}(x) u^{\frac{N k(2 N-3)}{2(N-2)}} d x \\
\leq C_{s}^{\frac{2 N(1-\theta)}{N-2}}\left(\int_{\Omega} a(x) u^{N k} d x\right)^{\theta}\left(\int_{\Omega}\left|\nabla a^{\frac{1}{2}}(x) u^{\frac{N k+m-1}{2}}\right|^{2} d x\right)^{\frac{N(1-\theta)}{N-2}} \\
\leq C_{s}^{\frac{2 N(1-\theta)}{N-2}}\left(\int_{\Omega} a(x) u^{N k} d x\right)^{\theta} \\
\quad \times\left[\frac{|A|}{2}\left(\int_{\Omega} a(x) u^{N k+m-1} d x\right)^{\frac{1}{2}}+\left(\int_{\Omega} a(x)\left|\nabla u^{\frac{N k+m-1}{2}}\right|^{2} d x\right)^{\frac{1}{2}}\right]^{\frac{2 N(1-\theta)}{N-2}} .
\end{aligned}
$$

Next, applying the inequality

$$
(x+y)^{k} \leq(2 \max \{x, y\})^{k} \leq 2^{k} \max \left\{x^{k}, y^{k}\right\} \leq 2^{k}\left(x^{k}+y^{k}\right), \quad \forall x, y, k \geq 0,
$$

and (31), we derive an estimate for the summation of the bracket in (37) as follows:

$$
\begin{aligned}
& {\left[\frac{|A|}{2}\left(\int_{\Omega} a(x) u^{N k+m-1} d x\right)^{\frac{1}{2}}+\left(\int_{\Omega} a(x)\left|\nabla u^{\frac{N k+m-1}{2}}\right|^{2} d x\right)^{\frac{1}{2}}\right]^{\frac{2 N(1-\theta)}{N-2}}} \\
& \quad \leq|A|^{\frac{2 N(1-\theta)}{N-2}}\left(\int_{\Omega}(a(x))^{\sigma_{1}} d x\right)^{\frac{N(1-\theta)(1-\gamma)}{N-2}}\left(\int_{\Omega} a^{\frac{N-2 \theta}{N-2}}(x) u^{\frac{N k(2 N-3)}{2(N-2)}} d x\right)^{\frac{N(1-\theta) \gamma}{N-2}} \\
& \quad+2^{\frac{2 N(1-\theta)}{N-2}}\left(\int_{\Omega} a(x)\left|\nabla u^{\frac{N k+m-1}{2}}\right|^{2} d x\right)^{\frac{N(1-\theta)}{N-2}} .
\end{aligned}
$$

Substituting (38) into (37), we have

$$
\begin{aligned}
\int_{\Omega} a^{\frac{N-2 \theta}{N-2}}(x) u^{\frac{N k(2 N-3)}{2(N-2)}} d x \\
\leq C_{4}\left(\int_{\Omega} a(x) u^{N k} d x\right)^{\theta}\left(\int_{\Omega} a^{\frac{N-2 \theta}{N-2}}(x) u^{\frac{N k(2 N-3)}{2(N-2)}} d x\right)^{\frac{N(1-\theta) \gamma}{N-2}} \\
\quad+C_{5}\left(\int_{\Omega} a(x) u^{N k} d x\right)^{\theta}\left(\int_{\Omega} a(x)\left|\nabla u^{\frac{N k+m-1}{2}}\right|^{2} d x\right)^{\frac{N(1-\theta)}{N-2}}
\end{aligned}
$$

where $C_{4}=\left(C_{s}|A|\right)^{\frac{2 N(1-\theta)}{N-2}}\left(\int_{\Omega}(a(x))^{\sigma_{1}} d x\right)^{\frac{N(1-\theta)(1-\gamma)}{N-2}}, C_{5}=\left(2 C_{s}\right)^{\frac{2 N(1-\theta)}{N-2}}$.

Now, applying Young's inequality to the two terms on the right-hand side of (39), we can get

$$
\begin{aligned}
& \left(\int_{\Omega} a(x) u^{N k} d x\right)^{\theta}\left(\int_{\Omega} a^{\frac{N-2 \theta}{N-2}}(x) u^{\frac{N k(2 N-3)}{2(N-2)}} d x\right)^{\frac{N(1-\theta) \gamma}{N-2}} \\
& \leq \frac{N-2-N(1-\theta) \gamma}{N-2} \varepsilon_{3}^{-\frac{N(1-\theta) \gamma}{N-2-N(1-\theta) \gamma}}\left(\int_{\Omega} a(x) u^{N k} d x\right)^{\frac{(N-2) \theta}{N-2-N(1-\theta) \gamma}} \\
& \quad+\frac{N(1-\theta) \gamma \varepsilon_{3}}{N-2} \int_{\Omega} a^{\frac{N-2 \theta}{N-2}}(x) u^{\frac{N k(2 N-3)}{2(N-2)}} d x
\end{aligned}
$$


and

$$
\begin{aligned}
& \left(\int_{\Omega} a(x) u^{N k} d x\right)^{\theta}\left(\int_{\Omega} a(x)\left|\nabla u^{\frac{N k+m-1}{2}}\right|^{2} d x\right)^{\frac{N(1-\theta)}{N-2}} \\
& \leq \frac{N-2-N(1-\theta)}{N-2} \varepsilon_{4}^{-\frac{N(1-\theta)}{N-2-N(1-\theta)}}\left(\int_{\Omega} a(x) u^{N k} d x\right)^{\frac{(N-2) \theta}{N-2-N(1-\theta)}} \\
& \quad+\frac{N(1-\theta) \varepsilon_{4}}{N-2} \int_{\Omega} a(x)\left|\nabla u^{\frac{N k+m-1}{2}}\right|^{2} d x
\end{aligned}
$$

where $\varepsilon_{3}, \varepsilon_{4}>0$ are constants to be determined later. Substituting (40), (41) into (39) leads to

$$
\begin{aligned}
(1- & \left.\frac{C_{4} N(1-\theta) \gamma \varepsilon_{3}}{N-2}\right) \int_{\Omega} a^{\frac{N-2 \theta}{N-2}}(x) u^{\frac{N k(2 N-3)}{2(N-2)}} d x \\
\leq & \frac{N-2-N(1-\theta) \gamma}{N-2} C_{4} \varepsilon_{3}^{-\frac{N(1-\theta) \gamma}{N-2-N(1-\theta) \gamma}}\left(\int_{\Omega} a(x) u^{N k} d x\right)^{\frac{(N-2) \theta}{N-2-N(1-\theta) \gamma}} \\
& +\frac{N-2-N(1-\theta)}{N-2} C_{5} \varepsilon_{4}^{-\frac{N(1-\theta)}{N-2-N(1-\theta)}}\left(\int_{\Omega} a(x) u^{N k} d x\right)^{\frac{(N-2) \theta}{N-2-N(1-\theta)}} \\
& +\frac{C_{5} N(1-\theta) \varepsilon_{4}}{N-2} \int_{\Omega} a(x)\left|\nabla u^{\frac{N k+m-1}{2}}\right|^{2} d x .
\end{aligned}
$$

Choose $\varepsilon_{3}>0$ small enough such that $\rho:=1-\frac{C_{4} N(1-\theta) \gamma \varepsilon_{3}}{N-2}>0$.

It follows that the second term on the right-hand side of (34) satisfies

$$
\begin{aligned}
& \int_{\Omega} a^{\frac{N-2 \theta}{N-2}}(x) u^{\frac{N k(2 N-3)}{2(N-2)}} d x \\
& \leq C_{6}\left(\int_{\Omega} a(x) u^{N k} d x\right)^{\frac{(N-2) \theta}{N-2-N(1-\theta) \gamma}}+C_{7}\left(\int_{\Omega} a(x) u^{N k} d x\right)^{\frac{(N-2) \theta}{N-2-N(1-\theta)}} \\
& \quad+C_{8} \int_{\Omega} a(x)\left|\nabla u^{\frac{N k+m-1}{2}}\right|^{2} d x
\end{aligned}
$$

where

$$
\begin{aligned}
& C_{6}=\frac{N-2-N(1-\theta) \gamma}{(N-2) \rho} C_{4} \varepsilon_{3}^{-\frac{\gamma N(1-\theta)}{N-2-\gamma N(1-\theta)}}, \\
& C_{7}=C_{5} \frac{N-2-N(1-\theta)}{(N-2) \rho} \varepsilon_{4}^{\frac{N(\theta-1)}{N-2-N(1-\theta)}}, \\
& C_{8}=\frac{C_{5} N(1-\theta) \varepsilon_{4}}{(N-2) \rho} .
\end{aligned}
$$

Then, substituting (42) into (44), we can derive

$$
\begin{aligned}
\psi_{3}^{\prime}(t) \leq & \left(C_{1}+C_{2} C_{8}\right) \int_{\Omega} a(x)\left|\nabla u^{\frac{N k+m-1}{2}}\right|^{2} d x \\
& +C_{2} C_{6} \psi_{3}^{\frac{(N-2) \theta}{N-2-N(1-\theta) \gamma}}+C_{2} C_{7} \psi_{3}^{\frac{(N-2) \theta}{N-2-N(1-\theta)}}+C_{3} .
\end{aligned}
$$


Choose $\varepsilon_{1}$ small enough such that $C_{1}<0$ and $\varepsilon_{4}$ such that $C_{1}+C_{2} C_{8}=0$. Therefore, (43) can be rewritten as

$$
\psi_{3}^{\prime}(t) \leq H_{3} \psi_{3}^{\frac{(N-2) \theta}{N-2-N(1-\theta) \gamma}}+H_{4} \psi_{3}^{\frac{(N-2) \theta}{N-2-N(1-\theta)}}+H_{5}
$$

where $H_{3}=C_{2} C_{6}, H_{4}=C_{2} C_{7}, H_{5}=C_{3}$.

Note that $\frac{(N-2) \theta}{N-2-N(1-\theta)}>1$, then integrating (44) from 0 to $t^{*}$, we derive

$$
t^{*} \geq \int_{\psi_{3}(0)}^{\infty} \frac{d \eta}{H_{3} \eta^{\frac{(N-2) \theta}{N-2-N(1-\theta) \gamma}}+H_{4} \eta^{\frac{(N-2) \theta}{N-2-N(1-\theta)}}+H_{5}} .
$$

Remark 3 If the null Dirichlet boundary condition (2) is replaced by the null Neumann boundary condition

$$
\frac{\partial u}{\partial v}(x, t)=0, \quad(x, t) \in \partial \Omega \times\left(0, t^{*}\right)
$$

where $v$ is the unit outward normal vector on $\partial \Omega$, then Theorem 1 is valid for the case $m \geq 1$, and Theorems $4-6$ are also valid.

\section{Conclusion}

Bounds for the blow-up time to a porous medium equation with weighted nonlocal source and inner absorption terms under some appropriate measure in the whole-dimensional space $(N \geq 1)$ are derived in this paper. Note that the methods for a semilinear parabolic equation in $[16,28]$ are not necessarily applicable to our quasilinear parabolic model, and our results extend the results for the model in [20,28].

\section{Acknowledgements}

The authors would like to deeply thank all the reviewers for their insightful and constructive comments.

\section{Funding}

Yunde Shen was supported by the project of Zhejiang Natural Science Foundation of China under (Grant No. LY16E050006).

\section{Abbreviations}

Not applicable.

Availability of data and materials

Data sharing not applicable to this article as no datasets were generated or analysed during the current study.

Competing interests

The authors declare that there is no conflict of interests regarding the publication of this paper.

Authors' contributions

All authors contributed equally and significantly in writing this article. All authors read and approved the final manuscript.

Author details

'School of College of Mechanical Electrical Engineering, Wenzhou University, Wenzhou, P.R. China. ${ }^{2}$ School of Mathematical Sciences, Ocean University of China, Qingdao, P.R. China.

\section{Publisher's Note}

Springer Nature remains neutral with regard to jurisdictional claims in published maps and institutional affiliations. 


\section{References}

1. Bebernes, J., Bressan, A.: Thermal behavior for a confined reactive gas. J. Differ. Equ. 44(1), 118-133 (1982)

2. Bebernes, J., Eberly, D.: Mathematical Problems from Combustion Theory. Springer, New York (1989)

3. Furter, J., Grinfield, M.: Local vs. nonlocal interactions in populations dynamics. J. Math. Biol. 27(1), 65-80 (1989)

4. Lee, C.B.: Possible universal transitional scenario in a flat plate boundary layer: measurement and visualization. Phys. Rev. E 62(3), 3659-3670 (2000)

5. Lee, C.B., Wu, J.Z.: Transition in wall-bounded flows. Appl. Mech. Rev. 61, 030802 (2008)

6. Lee, C.B., Peng, H.W., Yuan, H.J., Wu, J.Z., Zhou, M.D., Hussain, F.: Experimental studies of surface waves inside a cylindrical container. J. Fluid Mech. 677, 39-62 (2011)

7. Samarskii, A.A., Galaktionov, V.A., Kurdyumov, S.P., Mikhailov, A.P.: Blow-up in Quasilinear Parabolic Equations. de Gruyter, Berlin (1995)

8. Vazquez, J.L.: The Porous Medium Equations: Mathematical Theory. Oxford University Press, Oxford (2007)

9. Quittner, R., Souplet, P.: Superlinear Parabolic Problems: Blow-up, Global Existence and Steady States. Birkhäuser, Basel (2007)

10. Galaktionov, V.A., Vazquez, J.L.: The problem of blow up in nonlinear parabolic equations. Discrete Contin. Dyn. Syst. 8(2), 399-433 (2000)

11. Levine, H.A.: Nonexistence of global weak solutions to some properly and improperly posed problems of mathematical physics: the method of unbounded Fourier coefficients. Math. Ann. 214(3), $205-220$ (1975)

12. Payne, L.E., Schaefer, S.W.: Lower bounds for blow-up time in parabolic problems under Neumann conditions. Appl. Anal. 85(10), 1301-1311 (2006)

13. Payne, L.E., Schaefer, S.W.: Lower bounds for blow-up time in parabolic problems under Dirichlet conditions. J. Math Anal. Appl. 328(2), 1196-1205 (2007)

14. Viglialoro, G.: Explicit blow-up time for two porous medium problems with different reaction terms. In: Trends in Differential Equations and Applications. SEMA SIMAI Springer Series, vol. 8, pp. 147-167. Springer, Cham (2016)

15. Baghaei, K., Hesaaraki, M.: Lower bounds for the blow-up time in the higher-dimensional nonlinear divergence form parabolic equations. C. R. Math. Acad. Sci. Paris 351(19-20), 731-735 (2013)

16. Song, J.C.: Lower bounds for the blow-up time in a non-local reaction-diffusion problem. Appl. Math. Lett. 24(5), 793-796 (2011)

17. Liu, Y.: Lower bounds for the blow-up time in a non-local reaction diffusion problem under nonlinear boundary conditions. Math. Comput. Model. 57(3-4), 926-931 (2013)

18. Tang, G.S., Li, Y.F., Yang, X.T.: Lower bounds for the blow-up time of the nonlinear non-local reaction diffusion problems in $\mathbb{R}^{N}(N \geq 3)$. Bound. Value Probl. 2014, 265 (2014). https://doi.org/10.1186/s13661-014-0265-5

19. Liu, D.M., Mu, C.L., Xin, Q.: Lower bounds estimate for the blow-up time of a nonlinear nonlocal porous medium equation. Acta Math. Sci. 32B(3), 1206-1212 (2012)

20. Fang, Z.B., Yang, R., Chai, Y.: Lower bounds estimate for the blow-up time of a slow diffusion equation with nonlocal source and inner absorption. Math. Probl. Eng. 2014(2), 1-6 (2014)

21. Bao, A.G., Song, X.F.: Bounds for the blowup time of the solutions to quasi-linear parabolic problems. Z. Angew. Math. Phys. 65(1), 115-123 (2014)

22. Ahmed, I., Mu, C.L., Zheng, P., Zhang, F.C.: Blow-up and global existence for the non-local reaction diffusion problem with time dependent coefficient. Bound. Value Probl. 2013, 239 (2013). https://doi.org/10.1186/1687-2770-2013-239

23. Fang, Z.B., Wang, Y.X.: Blow-up analysis for a semilinear parabolic equation with time-dependent coefficients under nonlinear boundary flux. Z. Angew. Math. Phys. 66(5), 1-17 (2015)

24. Liu, Z.Q., Fang, Z.B.: Blow-up phenomena for a nonlocal quasilinear parabolic equation with time-dependent coefficients under nonlinear boundary flux. Discrete Contin. Dyn. Syst., Ser. B 21(10), 3619-3635 (2016)

25. Marras, M., Vernier Piro, S.: Explicit estimates for blow-up solutions to parabolic systems under nonlocal boundary conditions. C. R. Acad. Bulgare Sci. 67(4), 459-466 (2014)

26. Marras, M., Vernier Piro, S.: Blow-up time estimates in nonlocal reaction-diffusion systems under various boundary conditions. Bound. Value Probl. 2017, 2 (2017). https://doi.org/10.1186/s13661-016-0732-2

27. Fang, Z.B., Chai, Y.: Blow-up analysis for a quasilinear parabolic equation with inner absorption and nonlinear Neumann boundary condition. Abstr. Appl. Anal. 2014, Article ID 289245 (2014)

28. Song, X.F., LV, X.S.: Bounds for the blowup time and blowup rate estimates for a type of parabolic equations with weighted source. Appl. Math. Comput. 236(236), 78-92 (2014)

29. Ma, L.W., Fang, Z.B.: Blow-up phenomena for a semilinear parabolic equation with weighted inner absorption under nonlinear boundary flux. Math. Methods Appl. Sci. 40(1), 115-128 (2017)

30. Ma, L.W., Fang, Z.B.: Blow-up analysis for a reaction-diffusion equation with weighted nonlocal inner absorptions under nonlinear boundary flux. Nonlinear Anal., Real World Appl. 32, 338-354 (2016)

31. Ma, L.W., Fang, Z.B.: Blow-up analysis for a nonlocal reaction-diffusion equation with Robin boundary conditions. Taiwan. J. Math. 21(1), 131-150 (2017) 